\title{
Pharyngeal Stimulation in Head and Neck Cancer Patients with Dysphagia: Functional Outcomes and Transcranial Magnetic Stimulation Motor Evoked Potentials
}

\author{
Carol A Boliek ${ }^{1 *}$, Jennifer A Harris ${ }^{1}$, Daniel C Sadowski², Teresa Paslawski ${ }^{1}$, Kelvin E Jones ${ }^{3}$, Liu Shi Gan ${ }^{3}$, and Jana M Rieger ${ }^{1,4}$ \\ ${ }^{1}$ Department of Communication Sciences and Disorders, Faculty of Rehabilitation Medicine, University of Alberta, Edmonton, Alberta, Canada \\ ${ }^{2}$ Division of Gastroenterology, Faculty of Medicine and Dentistry, Edmonton, Alberta, Canada \\ ${ }^{3}$ Neuroscience and Mental Health Institute, University of Alberta, Edmonton, Alberta, Canada \\ ${ }^{4}$ Institute for Reconstructive Sciences in Medicine, Misericordia Hospital, Edmonton, Alberta, Canada
}

\section{Abstract}

Purpose: Head and neck cancer patients often experience swallowing disorders, impacting health and quality of life. This study examined effects of electrical stimulation to the pharynx on swallowing outcomes in post-surgical head and neck cancer patients.

Methods: Swallowing was assessed using video fluoroscopy before, and 30 minutes after, a ten-minute application of electrical stimulation to the pharynx in five patients experiencing moderate-severe dysphagia. Corticopharyngeal motor projections were measured before and after stimulation of the pharynx using transcranial magnetic stimulation of motor cortex and measurements of motor evoked potentials (MEPs) in the striated muscle of the posterior pharyngeal wall.

Results: Short-term changes in swallowing function were observed post-stimulation in: pharyngeal transit time, cricopharyngeal opening duration, total number of swallows, penetration/aspiration score, and duration of contact between the base of tongue and posterior pharyngeal wall. MEPs could not be measured in two participants. In the remaining participants, the MEP measures were not strongly associated with changes in observed swallowing function as has been found for neurogenic dysphagia.

Conclusions: These findings indicated that somatosensory input, generated by electrical stimulation of the pharynx, changes swallowing function in head and neck cancer patients. These changes are not strongly correlated with alterations of corticopharyngeal excitability as previously observed in acutely dysphagic stroke patients.

Keywords: Deglution; Deglutition disorders; Head and neck cancer; Transcranial magnetic stimulation; motor evoked potentials; Electrical stimulation of the pharynx

\section{Introduction}

Oncologic interventions for head and neck cancer may include radiation, chemotherapy, surgical removal of affected tissues, or some combination of these. As such, these patients are at risk for developing dysphagia and subsequent malnutrition and aspiration pneumonia. Dysphagia also has significant social implications because affected individuals limit their participation in social activities where eating is central [1].

Management options for swallowing disorders include: modification of diet texture, behavioural modifications, swallowing exercises, and use of enteral (tube) feeding. The application of electrical stimulation, both externally to the submental region and internally to the pharynx has been explored [2-4].

Hamdy et al. applied electrical stimulation to the pharyngeal muscles of healthy participants and found changes in cortical excitability, measured by transcranial magnetic stimulation (TMS) [3]. They noted an increase in motor cortex excitability in the cortical area representing the pharynx [3]. The same paradigms have been applied to dysphagic hemiplegic patients resulting in improved swallowing function [2]. Electrical stimulation of the pharyngeal wall may provide benefit to other populations with dysphagia, such as patients treated for head and neck cancer.

The purpose of the present study was to determine if electrical stimulation of sensory nerves in the pharynx in patients treated for head and neck cancer would result in improvement in the efficiency of bolus transport and clearance as well as the safety and effectiveness of bolus clearance. It was predicted that improvement in swallowing function would be observed following stimulation. A secondary goal of the study was to determine: 1) if TMS evoked MEPs could be measured in the pharyngeal muscles of head and neck cancer patients and, 2) if improvements in swallowing function would be associated with increased excitability of motor cortex projections to these muscles.

\section{Materials and Methods}

\section{Participants}

Five male participants treated with primary surgery and radial forearm free flap reconstruction with adjuvant radiation therapy for oropharyngeal cancer were recruited for this pre-post treatment study. All participants gave informed consent as approved by the Human Research Ethics Board at the University of Alberta. The Transcranial Magnetic Stimulation Adult Safety Screen (TASS) [6] was administered to each participant. Table 1 shows the participant demographic information, oncological treatment, and swallowing status. To be included, participants had to be diagnosed with chronic dysphagia involving the pharyngeal stage of the swallow. Additionally, all participants had an indication within their operative report that

*Corresponding author: Carol A Boliek, Department of Communication Sciences and Disorders, 2-70 Corbett Hall, Faculty of Rehabilitation Medicine, University of Alberta, Canada, T6G 2G4, Tel: 780-492-0841; E-mail: carol.boliek@ualberta.ca

Received October 13, 2014; Accepted December 30, 2014; Published January 10, 2015

Citation: Boliek CA, Harris JA, Sadowski DC, Paslawski T, Jones KE, et al (2015) Pharyngeal Stimulation in Head and Neck Cancer Patients with Dysphagia Functional Outcomes and Transcranial Magnetic Stimulation Motor Evoked Potentials. Int J Neurorehabilitation 2: 138. doi:10.4172/2376-0281.1000138

Copyright: $\odot 2015$ Boliek CA, et al. This is an open-access article distributed under the terms of the Creative Commons Attribution License, which permits unrestricted use, distribution, and reproduction in any medium, provided the original author and source are credited. 
Citation: Boliek CA, Harris JA, Sadowski DC, Paslawski T, Jones KE, et al. (2015) Pharyngeal Stimulation in Head and Neck Cancer Patients with Dysphagia: Functional Outcomes and Transcranial Magnetic Stimulation Motor Evoked Potentials. Int J Neurorehabilitation 2: 138. doi:10.4172/2376-0281.1000138

Page 2 of 6

some native tissue remained in the posterior pharyngeal wall of the oropharynx. All participants were at least twelve months post-surgery at the time of recruitment.

\section{Instrumentation}

A standard video fluoroscopic unit was used to acquire pre- and poststimulation swallowing studies, recorded to super-VHS video-recording media and transferred to a KayPENTAX Digital Swallowing Workstation (KayPENTAX, Lincoln Park, NJ, U.S.A.) for analyses of swallowing events.

Transcranial magnetic stimulation (TMS) of the cortex was delivered via a circular coil (outer diameter $=70 \mathrm{~mm}$; maximum output of 2.2 Tesla) connected to a magnetic stimulator set at single monophasic pulse (Magstim 200², The Magstim Company Ltd., Whitland, Carmarthenshire, UK). Motor evoked potentials (MEPs) elicited by the TMS were recorded from the pharyngeal muscles via a $3 \mathrm{~mm}$ transnasal catheter (Gaeltec Ltd, Dunvegan, Isle of Skye, Scotland). The catheter housed two biopolar platinum ring electrode pairs (Gaeltec, Dunvegan, Scotland) positioned 5 and $12 \mathrm{~cm}$ from the tip of the catheter with an interelectrode distance (within each electrode pair) of $1 \mathrm{~cm}$. Midway between the electrode pairs was a solid-state strain-gauge transducer used to assess pressure at the level of the cricopharyngeus sphincter.

The electrodes were connected to a preamplifier [filter settings; $5 \mathrm{~Hz}$ - 2 kHz (CED 1902, Cambridge Electronic Design, Cambridge, UK)]. A laboratory interface (Micro 1401 plus, Cambridge Electronic Design, Cambridge, UK) was used to collect pharyngeal MEPs (sampling rate of $4-8 \mathrm{kHz}$ ). Pharyngeal response data were recorded on a computer using custom scripts (SPIKE 2 software, v-6.0, Cambridge Electronic Design, Cambridge, UK).

Electrical stimulation of the posterior pharyngeal wall was administered using the same trans-nasal catheter described above. The catheter was connected to an electrical stimulator (Digitimer DS7A Constant Current Stimulator, Digitimer Ltd., Welwyn Garden City, Hertfordshire, UK). Stimulation parameters $(5 \mathrm{~Hz}$, pulse duration $0.2 \mathrm{~ms}$ ) were set using custom scripts (SPIKE 2 software, version 6.0, Cambridge Electronic Design, Cambridge, UK).

\section{Procedures}

Prior to stimulation, a video fluoroscopic swallowing study (VFSS) was administered. Two recordings of each patient's swallows were attempted for three consistencies: liquid (water mixed with liquid barium [Polibar Plus Liquid, barium sulfate suspension, Therapex] in a 3:1 ratio), pudding (approximately $10 \mathrm{cc}$ mixed with barium paste [Esobar, barium sulfate cream, Therapex] in a 3:1 ratio presented as a bolus on a teaspoon), and cookie (1/4 of a Digestive cookie with barium paste). Not all consistencies were trialed with every participant based on patient safety.

After the pre-stimulation VFSS, a pharyngeal catheter was inserted trans-nasally on the side that had native pharyngeal tissue remaining, using anesthetic lidocaine gel. The catheter was positioned relative to the cricopharyngeus and remained in-situ for ten minutes before starting the stimulation protocol to allow for habituation.

In preparation for the TMS protocol, the cranial vertex and approximate area of pharyngeal cortex were marked on the scalp $[7,8]$. The cortical site evoking the largest MEPs was determined and marked, starting with the hemisphere contralateral to the side of the pharynx with the most damage. A stimulus response curve was obtained for each hemisphere at the site found to evoke the largest MEPs. This was done beginning with the stimulator output that evoked the largest MEPs then decreased by $5 \%$ increments until an operationally defined threshold was reached (an MEP less than $20 \mu \mathrm{V}$ evoked in 2/5T MS stimuli).
Ten minutes of electrical stimulation $(5 \mathrm{~Hz}$, pulse duration 0.2 ms) were administered to the pharynx following a standardized stimulation protocol [2]. Pharyngeal sensory thresholds (ST) and maximum intensity tolerated were ascertained over 5 trials and used to determine the intensity of the treatment electrical stimulation [9]. In one case, (S1) stimulation was set to the maximum deliverable intensity of the stimulator, as the maximal tolerable stimulus intensity was not reached. Treatment intensity of electrical stimulation was calculated using the following formula: intensity $=$ AveSTmin +0.75 (AveSTmax - AveSTmin), where AveSTmax was the maximum intensity tolerated and AveST min was the lowest intensity that the patient could sense. One participant (S3) could not tolerate this stimulation intensity; the amplitude was dropped to: AveSTmin + 0.50 (AveSTmin - AveSTmin).

Immediately following stimulation, MEP response curves were captured using TMS over the same pre-stimulation sites. A video fluoroscopic swallowing study was administered using consistencies trialed pre-stimulation. Maximum facilitation effects with $5 \mathrm{~Hz}$ stimulation have been found at 30 minutes post-stimulation [2] thus, all video fluoroscopic exams were completed within 30 minutes of stimulation.

\section{Data measurement and analysis}

Videofluoroscopic footage was analyzed by a clinician blinded to the condition (i.e., pre- or post- stimulation). Variables characterizing the efficiency of bolus transport were durational in nature. These included pharyngeal transit time, swallowing response time, cricopharyngeal opening duration, and oral transit time and were measured based on methods reported in previous research [10]. Duration was not calculated for liquid boluses due to extraneous head movements associated with retrieving small volumes from a cup. The efficiency of bolus clearance was assessed by judging variables related to swallow residue. These included pharyngeal residue, which was rated on a 3-point scale described elsewhere [10], and the total number of swallows required to clear one bolus. The safety and effectiveness of bolus clearance was evaluated by assessing variables related to the protection of the airway and propulsion of the bolus through the pharynx. These included grading penetration/aspiration using the Penetration/Aspiration Scale [11], assessing Base of tongue-posterior pharyngeal wall (BOT-PPW) contact (yes/no), measuring duration of BOT-PPW contact with the posterior pharyngeal wall (from first point of any contact to last), and noting epiglottic inversion (yes/no). The total number of opportunities for post-stimulus changes was calculated separately for each of the three categories. Then, the actual number of changes observed within each category was divided by the total and multiplied by 100 to obtain a measure of percent change following stimulation.

To establish inter-rater reliability on all swallowing variables, another rater evaluated $20 \%$ of the video fluoroscopic data. The intra class correlation coefficient was 0.977 indicating strong inter-rater reliability.

To determine whether potential pre- to post-stimulation differences found were clinically relevant as opposed to a reflection of normal variance, archived video fluoroscopic footage collected from 16 patients with oropharyngeal cancer who had undergone surgery and associated oncological treatments for their disease were used as comparators to the present sample. Normal variance between two successive boluses of pudding in the same patient for all 16 patients was used to derive a set of comparison data. The difference scores for all 16 patients were ordered from the smallest to largest for each variable. Then a value of $70 \%$ (i.e., the mean difference value of the $11^{\text {th }}$ patient for each variable and 1.5 standard deviation bandwidth) was used to represent the majority of values.

Raw signals containing a MEP were averaged ( $n=3-5$ trials) for each 
Citation: Boliek CA, Harris JA, Sadowski DC, Paslawski T, Jones KE, et al. (2015) Pharyngeal Stimulation in Head and Neck Cancer Patients with Dysphagia: Functional Outcomes and Transcranial Magnetic Stimulation Motor Evoked Potentials. Int J Neurorehabilitation 2: 138. doi:10.4172/2376-0281.1000138

Page 3 of 6

\begin{tabular}{|c|c|c|c|c|c|c|c|c|}
\hline Participant & $\mathrm{Age}^{*}$ & Cancer Dx & Surgery (resected tissue) & Time post Sx (months) ${ }^{*}$ & Radiation & Chemo-therapy & Enteral Feeding & Oral diet ${ }^{*}$ \\
\hline S1 & 70 & T2 SCC & 75\% SP; R LPW; 50\% PPW & 23 & $\checkmark$ & $\checkmark$ & $\checkmark$ & Jell-O \\
\hline S2 & 55 & T3 SCC & $\begin{array}{l}\text { 75\% R BOT; R tonsil; } \\
\text { R LPW; 50\% R tongue }\end{array}$ & 19 & $\checkmark$ & $\checkmark$ & $\checkmark$ & $\begin{array}{l}\text { Thin liquids } \\
\text { Apple sauce }\end{array}$ \\
\hline s3 & 52 & T3 SCC & $50 \%$ BOT; $100 \%$ SP; LPW & 24 & $\checkmark$ & - & - & $\begin{array}{c}\text { Solids } \\
\text { Thin liquids }\end{array}$ \\
\hline S4 & 55 & T2 SCC & 25\% BOT; 33\% SP; L LPW & 12 & $\checkmark$ & $\checkmark$ & - & $\begin{array}{l}\text { Soft solids } \\
\text { Thin liquids }\end{array}$ \\
\hline S5 & 75 & T3 SCC & 100\% BOT; 25\% SP; R LPW; R tonsil & 32 & $\checkmark$ & $\checkmark$ & $\checkmark$ & Pudding consistency \\
\hline
\end{tabular}

*at time of study

$\mathrm{SCC}=$ squamous cell carcinoma; $\mathrm{SP}=$ soft palate $\mathrm{PS}=$ piriform sinus $; \mathrm{LPW}=$ lateral pharyngeal wall

$\mathrm{PPW}=$ posterior pharyngeal wall; $\mathrm{L}=$ left; $\mathrm{R}=$ right; $\mathrm{BOT}=$ base of tongue

$\mathrm{Dx}=$ diagnosis; $\mathrm{Sx}=$ Surgery; $\mathrm{SCC}=$ squamous cell carcinoma; $S P=$ soft palate; $P S=$ piriform sinus; $L P W=$ lateral pharyngeal wall; $P P=$ posterior pharyngeal wall; $L=l$ eft; $R=$ right BOT=base of tongue

Table 1: Demographic Information.

\begin{tabular}{|c|c|c|c|c|c|c|c|c|c|c|c|}
\hline & & S1 & S2 & \multicolumn{3}{|c|}{ S3 } & \multicolumn{2}{|c|}{ S4 } & \multicolumn{2}{|c|}{ S5 } & \multirow{2}{*}{$\begin{array}{c}{ }^{*} \text { Controls } \\
\text { Inter-swallow mean difference } \\
\text { P }\end{array}$} \\
\hline & & $\mathrm{P}$ & $\mathrm{L}$ & $\mathrm{L}$ & $\mathrm{P}$ & C & $\mathrm{L}$ & $\mathrm{P}$ & $\mathrm{L}$ & $\mathrm{P}$ & \\
\hline \multirow[b]{2}{*}{ OTT (sec) } & pre & 2.369 & & & 0.534 & & & 0.133 & & 0 & \multirow{2}{*}{0.334} \\
\hline & post & 6.006 & & & 0.267 & & & 0.267 & & 0.234 & \\
\hline \multirow{2}{*}{$\begin{array}{l}\text { SRT } \\
\text { (sec) }\end{array}$} & pre & 8.408 & & & 0.534 & & & 1.602 & & - & \multirow{2}{*}{0.383} \\
\hline & post & - & & & 0.400 & & & 1.735 & & - & \\
\hline \multirow{2}{*}{$\begin{array}{l}\text { PTT } \\
(\mathrm{sec})\end{array}$} & pre & 32.433 & & & 1.235 & & & 2.169 & & 1.502 & \multirow{2}{*}{0.601} \\
\hline & post & 9.610 & & & 0.934 & & & 1.735 & & 3.237 & \\
\hline \multirow{2}{*}{$\begin{array}{l}\text { COD } \\
(\mathrm{sec})\end{array}$} & pre & 0.801 & & & 0.534 & & & 0.567 & & 0.567 & \multirow{2}{*}{0.100} \\
\hline & post & 0.801 & & & 0.400 & & & 0.467 & & 0.434 & \\
\hline \multirow{2}{*}{$\begin{array}{l}\text { PR } \\
\text { (1 to 3) }\end{array}$} & pre & 3 & 3 & 3 & 3 & - & 2 & 1 & 3 & 3 & \multirow{2}{*}{0} \\
\hline & post & 3 & 3 & 3 & 3 & 1 & 1 & 1 & 3 & 3 & \\
\hline \multirow{2}{*}{$\begin{array}{l}\text { TNS } \\
(\#)\end{array}$} & pre & 8 & 7 & 4 & 18 & 1 & 2 & 2 & 3 & 6 & \multirow{2}{*}{1} \\
\hline & post & 6 & 6 & 7 & 13 & 8 & 2 & 2 & 4 & 3 & \\
\hline \multirow{2}{*}{$\begin{array}{l}\text { PAS } \\
\text { (0 to } 8)\end{array}$} & pre & 7 & 3 & 3 & 2 & - & 3 & 2 & 8 & 7 & \multirow{2}{*}{0} \\
\hline & post & 8 & 4 & 3 & 3 & 1 & 5 & 2 & 7 & 2 & \\
\hline \multirow{2}{*}{$\begin{array}{l}\text { BOT-PPW } \\
(\mathrm{Y} / \mathrm{N})\end{array}$} & pre & $Y$ & $\mathrm{~N}$ & $\mathrm{Y}$ & $Y$ & $Y$ & $Y$ & $Y$ & $\mathrm{~N}$ & $\mathrm{~N}$ & \multirow[b]{2}{*}{ NA } \\
\hline & post & $Y$ & $\mathrm{~N}$ & $\mathrm{Y}$ & $Y$ & $Y$ & $Y$ & $Y$ & $\mathrm{~N}$ & $\mathrm{~N}$ & \\
\hline \multirow{2}{*}{$\begin{array}{l}\text { DBP } \\
(\mathrm{sec})\end{array}$} & pre & 0.784 & & & 0.751 & & & 0.217 & & - & \multirow{2}{*}{0.167} \\
\hline & post & 1.051 & & & 0.918 & & & 0.517 & & - & \\
\hline \multirow{2}{*}{$\begin{array}{l}\mathrm{EI} \\
(\mathrm{Y} / \mathrm{N})\end{array}$} & pre & $\mathrm{N}$ & $\mathrm{N}$ & $\mathrm{N}$ & $\mathrm{N}$ & $\mathrm{N}$ & $\mathrm{N}$ & $Y$ & $\mathrm{~N}$ & $\mathrm{~N}$ & \multirow[b]{2}{*}{ NA } \\
\hline & post & $\mathrm{N}$ & $\mathrm{N}$ & $\mathrm{N}$ & $\mathrm{N}$ & $\mathrm{N}$ & $Y$ & $Y$ & $N$ & $\mathrm{~N}$ & \\
\hline
\end{tabular}

Caption: $L=$ liquid; $P=$ pudding; $C=$ cookie; $O T T=$ oral transit time; $S R T=$ swallowing response time; $P T T=$ pharyngeal transit time; $C O D=$ cricopharyngeal opening duration; $\mathrm{PR}=$ pharyngeal residue; TNS=total number of swallows; $\mathrm{PAS}=$ penetration/aspiration score; $\mathrm{BOT}-\mathrm{PPW}=$ base of tongue to post-pharyngeal wall contact; $\mathrm{DBP}=$ duration base of tongue to post-pharyngeal wall contact; El=epiglottic inversion. ${ }^{*}=$ swallow-to-swallow variability (in mean difference) observed in the majority of the comparison data; bolded cells show changes outside of the range of $70 \%$ of the comparison data (pudding only). Cells with (-) indicate data could not be measured for that participant.

Table 2: Descriptive data for patients (S1-S5) and controls.

TMS output level evoking a response. MEP averaging was done with a time window of $110 \mathrm{~ms}$ (10ms prior to the TMS pulse to $100 \mathrm{~ms}$ after the TMS pulse). The baseline reference for the MEP responses was set from EMG signals obtained 10ms pre-TMS pulse. Dependent measures from the MEPs included peak amplitude, onset latency, and MEP threshold.

Descriptive analyses were completed on all ten swallowing variables and MEPs. Due to the exploratory nature of this study and limited sample size, no statistical tests were run.

\section{Results}

Participant demographics are presented in Table 1. Swallowing outcomes are presented in Table 2. Four of the 5 participants showed pre-to-post-stimulation changes in swallowing. These changes fell outside the swallow-to-swallow variability derived from the comparative data. The two variables that showed the greatest change were cricopharyngeal opening duration (COD) and duration base of tongue to post-pharyngeal wall contact (DBP). Post-stimulation changes also were observed for pharyngeal transit time (PTT), total number of swallows (TNS), and penetration-aspiration score (PAS). As can be seen in Table 2, the direction of post-stimulation changes was not consistent across participants.

Participant one (S1) exhibited changes that fell outside the comparison swallow-to-swallow variability for five of the variables. Participant two (S2) did not exhibit change in any variable poststimulation. Participant four (S4) demonstrated two changes, while participants three and five (S3, S5) exhibited four changes each.

The total number of opportunities for post-stimulus changes was calculated separately for each cluster of variables representing bolus transport efficiency (OTT, SRT, PTT, COD), bolus clearance efficiency (PR, TNS) and safety and effectiveness of bolus clearance (PAS, BOTPPW, DBP, EI). There was a $30 \%$ change-rate for bolus transport efficiency. Bolus clearance efficiency showed a $17 \%$ change poststimulation. Safety and effectiveness of bolus clearance changed by $17 \%$. 
Citation: Boliek CA, Harris JA, Sadowski DC, Paslawski T, Jones KE, et al. (2015) Pharyngeal Stimulation in Head and Neck Cancer Patients with Dysphagia: Functional Outcomes and Transcranial Magnetic Stimulation Motor Evoked Potentials. Int J Neurorehabilitation 2: 138. doi:10.4172/2376-0281.1000138

Page 4 of 6

Prior to stimulation, MEPs were evoked in two of five participants (Figure 1). In a third participant (S3) MEPs were evoked only after stimulation. Participant S1 also showed more modest increases in
MEP amplitude. However, in another participant (S2, Figure 1) the MEP responses were consistent with a modest depression of corticopharyngeal motor projections after stimulation. MEPs could not
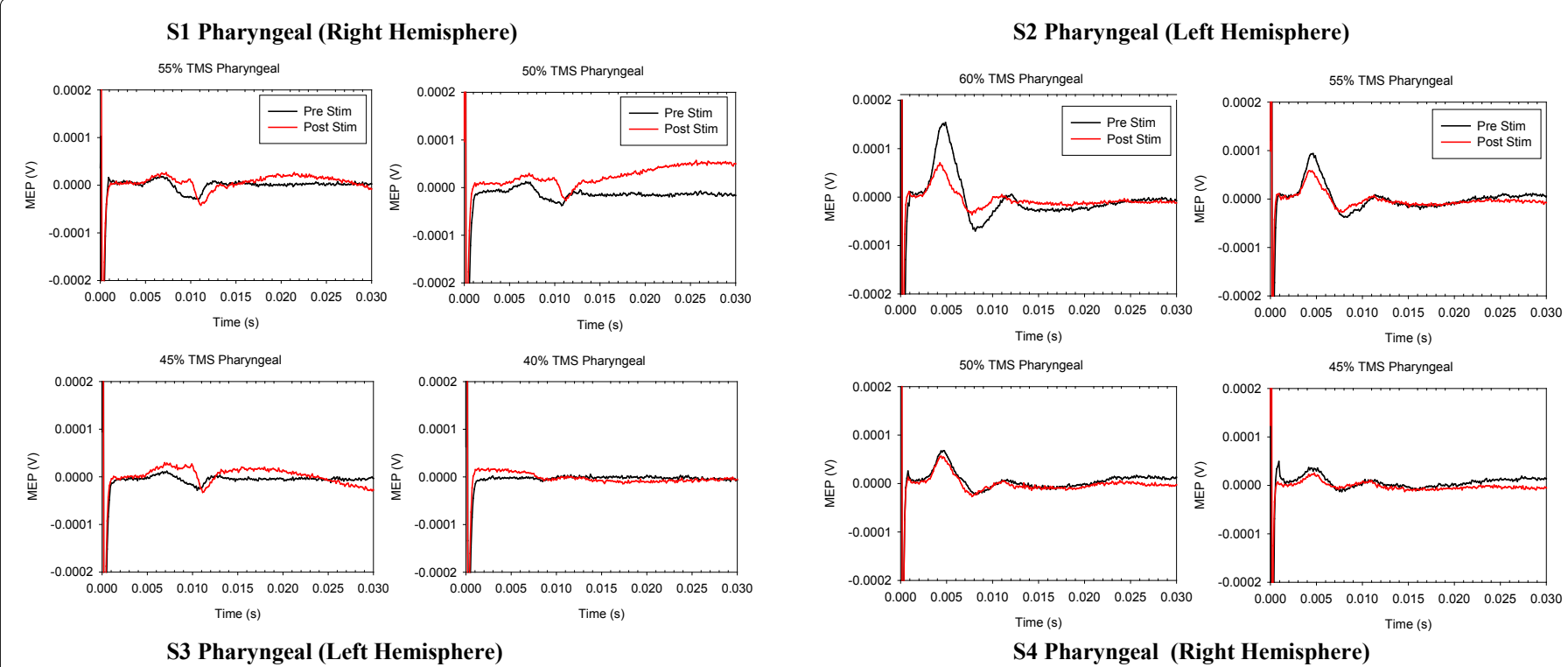

S3 Pharyngeal (Left Hemisphere)
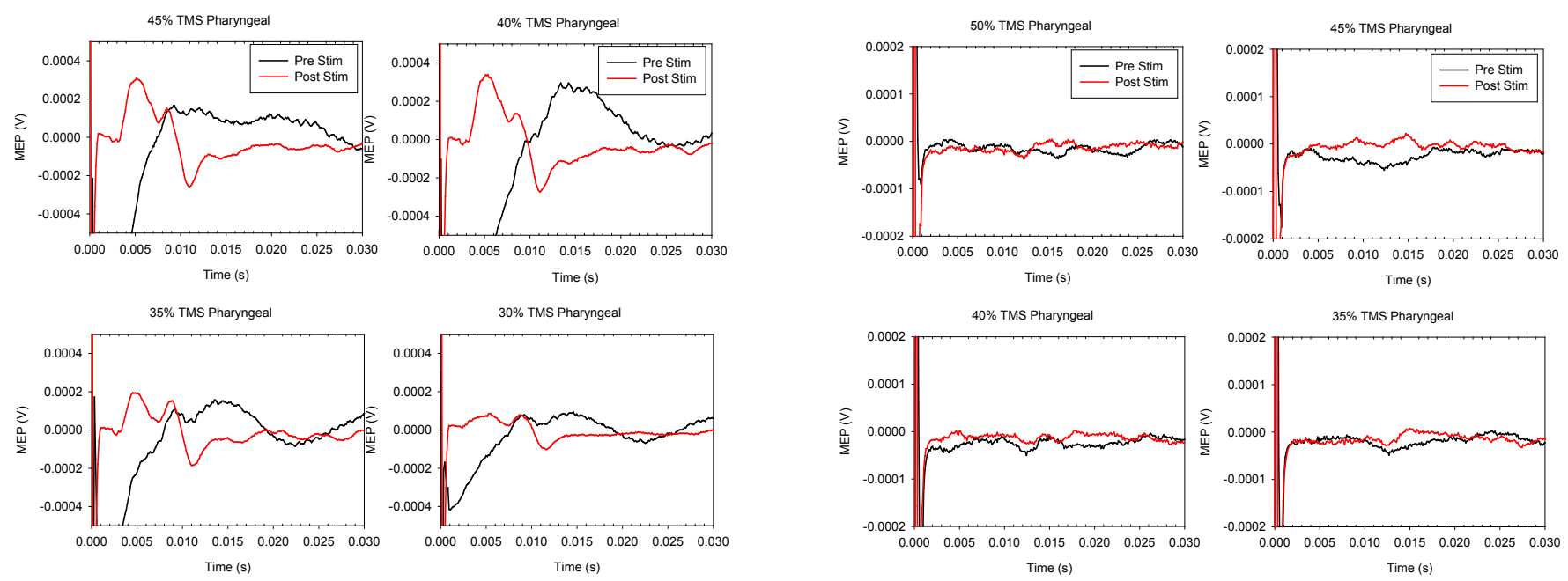

S5 Pharyngeal (Left Hemisphere)
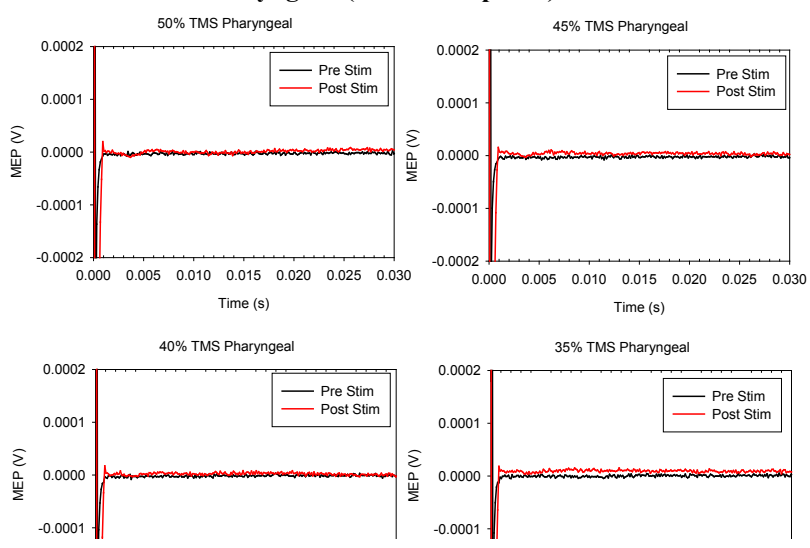

MEPs from TMS pulses delivered over the dominant swallowing cortex for S1- S5. Each panel depicts the TMS pulse strength used to elicit MEPs for each participant. For each panel, MEP amplitude is depicted on the $y$ axis in volts (V) and time following the TMS pulse burst is represented in second (sec) on the $x$ axis. MEP waveforms depicted in black were gathered pre-stimulation. MEP waveforms depicted in red represent post-stimulation. Note the black MEP waveform depicted for S3 is indicative of DC drift only. No MEPs were detected in participants S4 and S5.

Figure 1: Motor evoked potentials (MEPs) from the pharyngeal wall pre- and post-electrical stimulation. 
Citation: Boliek CA, Harris JA, Sadowski DC, Paslawski T, Jones KE, et al. (2015) Pharyngeal Stimulation in Head and Neck Cancer Patients with Dysphagia: Functional Outcomes and Transcranial Magnetic Stimulation Motor Evoked Potentials. Int J Neurorehabilitation 2: 138. doi:10.4172/2376-0281.1000138

Page 5 of 6

be evoked in the remaining two participants.

\section{Discussion}

The purpose of the present study was to determine whether functional improvement in the swallowing ability of patients with head and neck cancer could be promoted using a standardized protocol of pharyngeal stimulation. This was the first known study where pharyngeal wall stimulation was applied to a group of patients following surgical treatment and adjuvant radiation therapy for head and neck cancer. The goal of the study was to see if changes in swallowing parameters would be detected following post-electrical stimulation. If so, peripheral stimulation may ultimately serve as a priming technique for use prior to or during behavioural treatment, or as an intervention coupled to daily eating activities. A secondary goal was to determine if functional improvement in swallowing was associated with increased excitability of corticopharyngeal motor pathways assessed using TMS. While the results of this study are preliminary, they show encouraging trends.

All but one of the participants showed some change outside the range of comparison swallow-to-swallow variation. However, the changes were not consistent in terms of number or direction. In addition, there were no clear patterns related to patient variables that could explain similarities and differences in post-stimulation outcomes.

To characterize functional improvement in swallowing ability, we clustered variables representing bolus transport efficiency, bolus clearance efficiency, and safety and effectiveness of bolus clearance. The greatest changes were in the bolus transport efficiency with some trends having clinical meaning. Shorter pharyngeal transit duration could be clinically important because, when summed across a whole meal, the result would be overall shorter meal durations. Fatigue and social discomfort associated with longer meal durations are significant as they may contribute to decreased intake and subsequent weight loss [1]. Cricopharyngeal opening duration also decreased following stimulation, which theoretically could have either a positive or negative swallowing outcome. Shorter cricopharyngeal durations could shorten the overall length of each swallow. However, if the opening duration is not long enough to clear the bolus, then more swallows will be required, which effectively lengthens the overall swallowing duration. In addition, it is possible that a shorter opening duration, with subsequent pharyngeal residue, could lead to post-swallow aspiration. This was not the case in the current study.

Two possible mechanisms behind observed post-stimulation changes include electrical stimulation environment or the calculations used to derive bolus transport efficiency variables. First, due to the moist mucosa of the oropharyngeal structures, electrical stimulation likely reached beyond areas immediately surrounding the electrodes such as the suprahyoid musculature. If stimulation had an inhibitory effect on muscle contraction like that reported in other studies [2], then this could have led to a decrease in hyolaryngeal excursion and shortened cricopharyngeal opening duration. Second, a shortened cricopharyngeal opening duration could have resulted in a calculated decreased pharyngeal transit time simply because pharyngeal transit time was calculated from the point that marked the end of cricopharyngeal closing.

Variables that characterized bolus clearance appeared to be less affected by stimulation compared to those related to bolus transport efficiency. The only notable observation was a change in the total number of swallows, which increased in some situations and decreased in others. A decrease in total number of swallows indicating a more efficient swallow could be of benefit clinically. However, fewer swallows could be a concern in cases where pharyngeal residue is still a threat.
Variables that characterized safety and effectiveness of bolus clearance also were less affected by stimulation compared to those related to bolus transport efficiency. The duration of the base of tongue to posterior pharyngeal wall contact was most affected by stimulation providing support for the notion that sensorimotor control of the tongue base and/or the pharyngeal constrictors were facilitated by stimulation. Lengthening the duration of contact of the tongue base to the posterior pharyngeal wall is related to effective pharyngeal clearance [12]. Moreover, achieving sufficient duration of BOT-PPW contact is necessary for establishing adequate driving pressure to move the bolus through the pharynx [12]. A longer duration of BOT-PPW contact therefore suggests a stronger, more effective mechanism to propel the bolus into the cricoesophagus.

This study demonstrates for the first time, the feasibility of recording pharyngeal MEPs in individuals following surgical treatment and adjuvant radiation therapy for head and neck cancer. The ability to evoke and measure MEPs did not appear to be related to the severity of the surgical intervention (i.e., comparing nonresponse in S4 and S5 to the others). The absence of response in two individuals could be due to a topographical reorganization of motor cortex that we were unable to detect with the circular TMS coil methodology [14] or, partial denervation of the muscle of interest.

We assessed the relationship between changes in swallowing outcomes after electrical sensory stimulation of the pharynx and changes in MEPs. The hypothesis based on previous studies of dysphagia following stroke was that MEP amplitude would increase by about $60 \%$ following a $10 \mathrm{~min}$ treatment of $5 \mathrm{~Hz}$ stimulation and this would be correlated with positive changes in swallowing function [2, $3,9]$. Changes in two of three participants (e.g., S1, S3) were consistent with this hypothesis. However, in participant S2 the peak amplitude of the MEP at a TMS stimulus intensity of $60 \%$ was smaller after the sensory stimulation treatment but this individual did not show any associated negative changes in swallowing function. Moreover, functional changes were observed in patients where no MEPs could be detected (e.g., S5). Fraser et al. [2] found that the functional effects of pharyngeal stimulation were very sensitive to stimulus frequency. In healthy participants they found that stimulation was facilitative at $5 \mathrm{~Hz}$, but inhibitory at 10,20, and $40 \mathrm{~Hz}$. Due to the peripheral tissue damage in the participants in the present study, it is reasonable to propose that frequencies other than $5 \mathrm{~Hz}$ may produce a greater effect.

These preliminary data suggest that electrical stimulation of the posterior pharyngeal wall may invoke short-term changes in cortical or subcortical sensorimotor swallowing pathways and supports observations made previously [2, 3, 9,13]. Several neural mechanisms could be responsible for the post-stimulation changes observed in some of the swallowing variables. A proposed central mechanism suggests that sensory axons were stimulated and, in turn, recruited spinal motoneurons through a reflex pathway $[15,16]$. This mechanism would result in a greater number of muscle fibers contracting during the swallow and would account for some of the changes in timing and contact between structures observed thirty minutes after stimulation. Other research has demonstrated plasticity in the motor cortex following peripheral nerve stimulation and TMS $[17,18]$. These researchers suggested that plasticity occurred at a cortical level, rather than in spinal motoneurons. A reverse protocol involving CNS stimulation would be required to confirm a centrally driven neural mechanism responsible for the changes seen in the present data. Fraser and colleagues [2] used a reverse protocol and demonstrated that changes in sensory input can produce changes in the cortical representation of swallowing. 
Citation: Boliek CA, Harris JA, Sadowski DC, Paslawski T, Jones KE, et al. (2015) Pharyngeal Stimulation in Head and Neck Cancer Patients with Dysphagia: Functional Outcomes and Transcranial Magnetic Stimulation Motor Evoked Potentials. Int J Neurorehabilitation 2: 138. doi:10.4172/2376-0281.1000138

Our lack of detecting a consistent relationship between pre- and post-stimulation MEPs is consistent with previous research. Others have found both positive and negative relationships between treatmentrelated changes in pharyngeal MEPs and swallowing function in patients following stroke [19,20] and those with Parkinson disease [21]. The lack of consistent outcomes indicates the complexity of underlying neural mechanisms associated with adequate swallowing function [22]. Moreover, methodological factors, stimulation protocols, variation in muscle activation patterns and MEPs, and surface-level detection (sEMG) of MEPs from the pharynx, likely add to inconsistent findings across studies (see 22 for a comprehensive review).

Although the protocol for this study was successful in laying the foundation for future studies into a therapeutic treatment option for dysphagic head and neck cancer patients, some limitations need consideration. First, the video fluoroscopic footage was taken only laterally, therefore limiting observations to one plane. When analyzing tongue base and pharyngeal behaviour, biplanar footage would allow for assessment of both lateral and posterior pharyngeal wall movement/ contact. Second, participant numbers were small and a control group to assess a placebo effect (sham protocol) was not possible [2]. Third, we could not control surgically related variables such as the amount of native tissue remaining and its innervation. Because of this, there is no guarantee that stimulation was uniform across all 5 participants.

\section{Conclusions}

This study examined the effects of electrical stimulation to the pharynx on swallowing outcomes in post-surgical head and neck cancer patients. We assessed swallowing using video fluoroscopy before, and 30 minutes after, a ten-minute application of electrical stimulation to the pharynx in five patients experiencing moderate-severe dysphagia. The findings of the present study indicated that somatosensory input, generated by electrical stimulation of the pharynx, changed swallowing function in this population. However, these changes were not strongly correlated with alterations of cortico-pharyngeal excitability, indicating the complexity associated with the underlying neural mechanisms associated with swallowing.

\section{Acknowledgement}

This study was funded by a grant from the University Hospital Foundation, Edmonton, Canada.

\section{References}

1. Ekberg O, Hamdy S, Woisard V, Wuttge-Hannig A, Ortega P (2002) Social and psychological burden of dysphagia: its impact on diagnosis and treatment. Dysphagia 17: 139-146.

2. Fraser C, Power M, Hamdy S, Rothwell J, Hobday D, et al. (2002) Driving plasticity in human adult motor cortex is associated with improved motor function after brain injury. Neuron 34: 831-840.

3. Hamdy S, Aziz Q, Rothwell JC, Hobson A, Thompson DG (1998) Sensorimotor modulation of human cortical swallowing pathways. J Physiol 506 : 857-866.

4. Shaw GY, Sechtem PR, Searl J, Keller K, Rawi TA, et al. (2007) Transcutaneous neuromuscular electrical stimulation (VitalStim) curative therapy for severe dysphagia: myth or reality? Ann Otol Rhinol Laryngol 116: 36-44.

5. Rothwell JC, Thompson PD, Day BL, Boyd S, Marsden CD (1991) Stimulation of the human motor cortex through the scalp. Exp Physiol 76: 159-200.
6. Keel JC, Smith MJ, Wassermann EM (2001) A safety screening questionnaire for transcranial magnetic stimulation. Clin Neurophysiol 112: 720.

7. Jasper $\mathrm{HH}$ (1958) The 10-20 electrode system of the International Federation. Electroenceph Clin Neurophys10: 371-375.

8. Hamdy S, Aziz Q, Rothwell JC, Singh KD, Barlow J, et al. (1996) The cortical topography of human swallowing musculature in health and disease. Nat Med 2: $1217-1224$

9. Gow D, Hobson AR, Furlong P, Hamdy S (2004) Characterising the centra mechanisms of sensory modulation in human swallowing motor cortex. Clin Neurophysiol 115: 2382-2390.

10. Rieger J, Zalmanowitz J, Li S, Sytsanko A, Harris J, et al. (2007) Functional outcomes after surgical reconstruction of the base of tongue using the radial forearm free flap in patients with oropharyngeal carcinoma. Head \& Neck 29: 1024-1032.

11. Rosenbek JC, Robbins JA, Roecker EB, Coyle JL, Wood JL (1996) A penetration-aspiration scale. Dysphagia 11: 93-98.

12. Pauloski BR, Logemann JA (2000) Impact of tongue base and posterior pharyngeal wall biomechanics on pharyngeal clearance in irradiated postsurgical oral and oropharyngeal cancer patients. Head Neck 22: 120-131.

13. Hamdy S, Rothwell JC, Aziz Q, Singh KD, Thompson DG (1998) Long-term reorganization of human motor cortex driven by short-term sensory stimulation. Nat Neurosci 1: 64-68.

14. Aziz Q, Rothwell JC, Hamdy S, Barlow J, Thompson DG (1996) The topographic representation of esophageal motor function on the human cerebral cortex. Gastroentreology111: 855-862.

15. Collins DF (2007) Central contributions to contractions evoked by tetanic neuromuscular electrical stimulation. Exerc Sport Sci Rev 35: 102-109.

16. Dean JC, Yates LM, Collins DF (2008) Turning off the central contribution to contractions evoked by neuromuscular electrical stimulation. Muscle Nerve 38: 978-986.

17. Stefan K, Kunesch E, Cohen LG, Benecke R, Classen J (2000) Induction of plasticity in the human motor cortex by paired associative stimulation. Brain 123 Pt 3: 572-584.

18. Ridding MC, Brouwer B, Miles TS, Pitcher JB, Thompson PD (2000) Changes in muscle responses to stimulation of the motor cortex induced by peripheral nerve stimulation in human subjects. Exp Brain Res 131: 135-143.

19. Michou E, Mistry S, Jefferson S, Singh S, Rothwell J, et al. (2012) Targeting unlesioned pharyngeal motor cortex improves swallowing in healthy individuals and after dysphagic stroke. Gastroenterology 142: 29-38.

20. Michou E, Mistry S1, Jefferson S1, Tyrrell P2, Hamdy S3 (2014) Characterizing the mechanisms of central and peripheral forms of neurostimulation in chronic dysphagic stroke patients. Brain Stimul 7: 66-73.

21. Michou E, Hamdy S2, Harris M1, Vania A1, Dick J3, et al. (2014) Characterization of corticobulbar pharyngeal neurophysiology in dysphagic patients with Parkinson's disease. Clin Gastroenterol Hepatol 12: 2037-2045.

22. Macrae PR, Jones RD, Huckabee ML (2014) The effect of swallowing treatments on corticobulbar excitability: A review of transcranial magnetic stimulation induced motor evolked potentials. J Neuro Meth 233: 89-98. 